\title{
Supplementary Material on:
}

\section{Revisiting the Liquid-liquid Phase Behavior of $n$ -}

\section{Alkanes and Ethanol}

Sven Diekmann ${ }^{1}$, Esther Dederer ${ }^{1}$, Sven Charmeteau ${ }^{1}$, Sabine Wagenfeld ${ }^{1}$, Johannes Kiefer ${ }^{1}$, Wolffram Schröer ${ }^{2}$, and Bernd Rathke,

(1) Universität Bremen, Technische Thermodynamik, Badgasteiner Str. 1, 28359 Bremen, Germany,

*To whom correspondence should be addressed: Tel.: +49 421218 64772; fax: +49 421218 64771;

e-mail: rathke@uni-bremen.de

(2) Universität Bremen, FB2, Institut für Anorganische und Physikalische Chemie, Leobener Str. NWII, 28359 Bremen, Germany. 

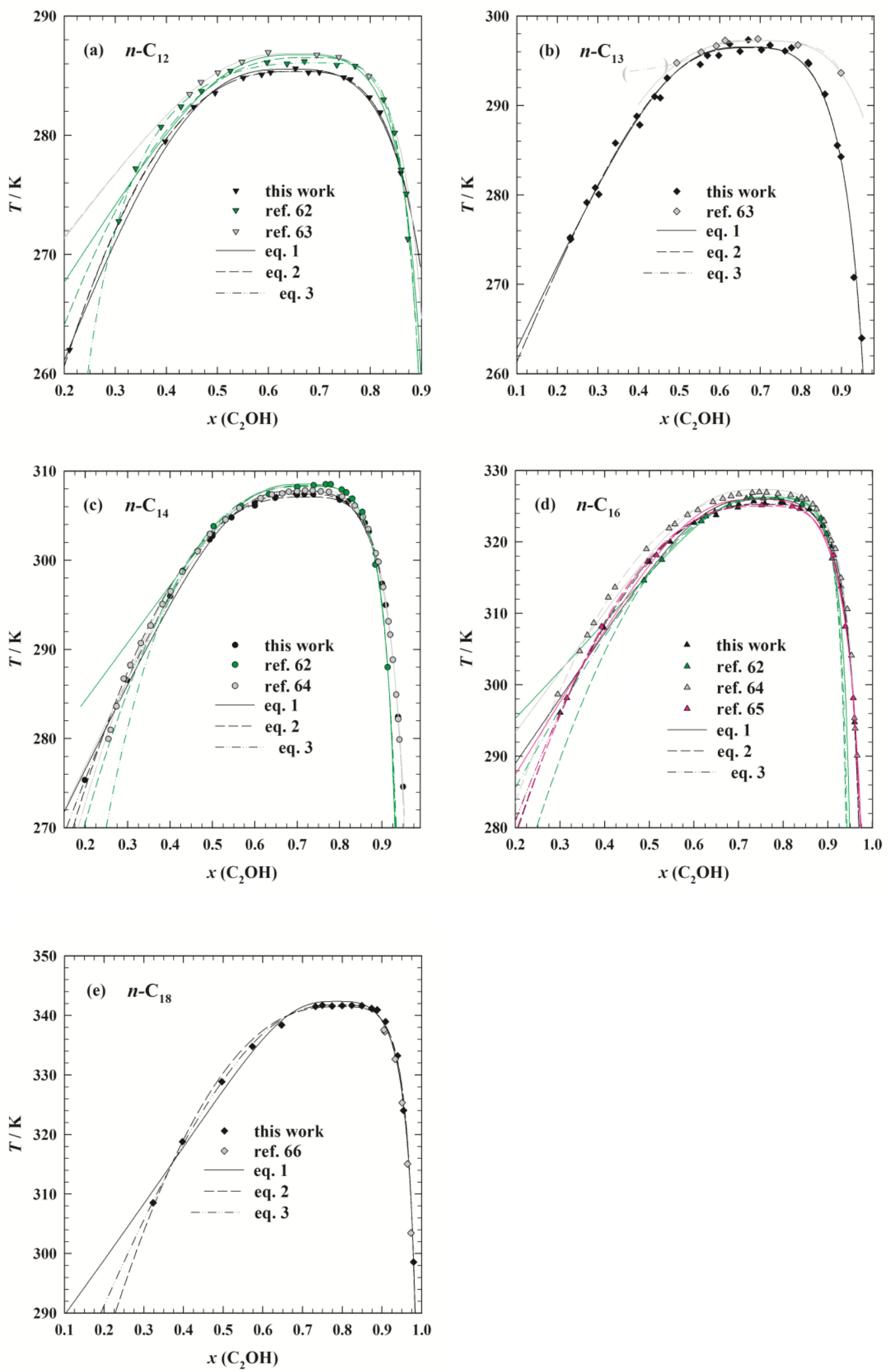
Figure S1: Isobaric phase diagrams at ambient pressure for ethanol with $n$-alkanes $\left(\mathrm{C}_{\mathrm{n}}\right)$ with the mole fraction $x$ of ethanol as composition variable as full symbols (this work). Data from literature are assigned by colored symbols. The sub-panels (a, b, c, d, e) depict data of $n$-alkanes with: (a): $n=12$; (b) $n=13$; (c) $n=14$, (d) $n=16$, and (e) $n=18$, each together with data available in literature: $n=12^{63,64} ; 13^{64}, 14^{63,65}, 16^{63,65,66}$, and $18 .{ }^{67}$ The lines indicate the fits with eq 1 (solid line), eq 2 (dashed line), and eq 3 (dashed/dotted line), which will be discussed in the next section. The numerical results of the fits are listed in Table 2. 
Table S1: Data set for the isobaric liquid-liquid phase diagrams at ambient pressure of ethanol - $n$-alkane mixtures; mass fractions $w_{\mathrm{Et}}$, mole fractions $x_{\mathrm{Et}}$ and cloud-point temperatures $T_{\text {cloud }}$ including their repeatability $\delta T$. The expanded uncertainty $(k=2)$ of the temperature measurement was $U(T) \leq \pm 0.25 \mathrm{~K}$. The uncertainty in the mole fraction is due to the accuracy of the weight measurement and the sample size $U\left(x_{\mathrm{IL}}\right)= \pm 10^{-4}$. Mixtures of ethanol and $n$-octane do show full miscibility at $T \geq 215 \mathrm{~K}$.

\begin{tabular}{|c|c|c|c|}
\hline \multicolumn{4}{|c|}{ ethanol + n-nonane } \\
\hline$w_{\mathrm{Et}}$ & $x_{\mathrm{Et}}$ & $T_{\text {cloud }} / \mathrm{K}$ & $\delta T / \mathrm{K}$ \\
\hline 0.1353 & 0.3034 & 237.72 & 0.05 \\
\hline 0.1608 & 0.3479 & 239.13 & 0.05 \\
\hline 0.1965 & 0.4051 & 240.94 & 0.05 \\
\hline 0.2258 & 0.4481 & 241.53 & 0.05 \\
\hline 0.2646 & 0.5004 & 241.75 & 0.05 \\
\hline 0.3070 & 0.5523 & 241.63 & 0.05 \\
\hline 0.3529 & 0.6029 & 241.64 & 0.05 \\
\hline 0.4013 & 0.6511 & 241.24 & 0.05 \\
\hline 0.4566 & 0.7005 & 240.13 & 0.05 \\
\hline
\end{tabular}

\begin{tabular}{|c|c|c|c|}
\hline \multicolumn{4}{|c|}{ ethanol $+\boldsymbol{n}$-decane } \\
\hline$w_{\mathrm{Et}}$ & $x_{\mathrm{Et}}$ & $T_{\text {cloud }} / \mathrm{K}$ & $\delta T / \mathrm{K}$ \\
\hline 0.0737 & 0.1973 & 239.51 & 0.13 \\
\hline 0.0967 & 0.2485 & 245.82 & 0.78 \\
\hline 0.1248 & 0.3058 & 252.25 & 0.90 \\
\hline 0.1767 & 0.3986 & 255.36 & 0.06 \\
\hline 0.2421 & 0.4967 & 257.07 & 0.07 \\
\hline
\end{tabular}




\begin{tabular}{|c|c|c|c|}
\hline 0.2823 & 0.5485 & 257.54 & 0.07 \\
\hline 0.3082 & 0.5791 & 257.68 & 0.05 \\
\hline 0.3271 & 0.6003 & 257.58 & 0.05 \\
\hline 0.3547 & 0.6293 & 257.45 & 0.05 \\
\hline 0.3713 & 0.6459 & 257.23 & 0.05 \\
\hline 0.4270 & 0.6972 & 256.70 & 0.10 \\
\hline 0.4913 & 0.7489 & 255.10 & 0.05 \\
\hline 0.5666 & 0.8015 & 252.05 & 0.55 \\
\hline 0.6459 & 0.8493 & 246.91 & 3.60 \\
\hline 0.6819 & 0.8688 & 240.37 & 1.20 \\
\hline
\end{tabular}

\begin{tabular}{|c|c|c|c|}
\hline \multicolumn{4}{|c|}{ ethanol + n-undecane } \\
\hline$w_{\text {Et }}$ & $x_{\mathrm{Et}}$ & $T_{\text {cloud }} / \mathrm{K}$ & $\delta T / \mathrm{K}$ \\
\hline 0.0714 & 0.2069 & 252.14 & 0.05 \\
\hline 0.1131 & 0.3020 & 262.91 & 0.05 \\
\hline 0.1672 & 0.4052 & 268.90 & 0.05 \\
\hline 0.1962 & 0.4531 & 271.25 & 0.05 \\
\hline 0.2682 & 0.5543 & 271.98 & 0.05 \\
\hline 0.3073 & 0.6008 & 272.45 & 0.05 \\
\hline 0.3522 & 0.6485 & 272.12 & 0.05 \\
\hline 0.4082 & 0.7006 & 271.89 & 0.05 \\
\hline 0.4824 & 0.7598 & 270.85 & 0.05 \\
\hline 0.5736 & 0.8203 & 266.18 & 0.05 \\
\hline 0.6442 & 0.8600 & 260.41 & 0.05 \\
\hline 0.7048 & 0.8901 & 252.32 & 0.05 \\
\hline 0.7483 & 0.9098 & 244.83 & 0.05 \\
\hline 0.7935 & 0.9288 & $242.26 * * S L E$ & 0.2 \\
\hline
\end{tabular}




\begin{tabular}{|l|l|l|l|}
\hline 0.8478 & 0.9497 & $241.72 * *$ SLE & 0.2 \\
\hline
\end{tabular}

\section{ethanol $+n$-dodecane}

\begin{tabular}{|c|c|c|c|}
\hline$w_{\mathrm{Et}}$ & $x_{\mathrm{Et}}$ & $T_{\text {cloud }} / \mathrm{K}$ & $\delta T / \mathrm{K}$ \\
\hline 0.0671 & 0.2100 & 262.01 & 0.05 \\
\hline 0.1516 & 0.3979 & 279.48 & 0.05 \\
\hline 0.1833 & 0.4535 & 282.37 & 0.05 \\
\hline
\end{tabular}

\begin{tabular}{|l|l|l|l|}
\hline 0.1833 & 0.4535 & 282.37 & 0.05 \\
\hline 0.2098 & 0.4954 & 283.55 & 0.05 \\
\hline
\end{tabular}

\begin{tabular}{|l|l|l|l|}
\hline 0.2489 & 0.5507 & 284.81 & 0.05 \\
\hline 0.2775 & 0.5868 & 285.09 & 0.05 \\
\hline 0.2924 & 0.6044 & 285.27 & 0.05 \\
\hline
\end{tabular}

\begin{tabular}{|l|l|l|l|}
\hline 0.2924 & 0.6044 & 285.27 & 0.05 \\
\hline 0.3373 & 0.6530 & 285.60 & 0.05 \\
\hline
\end{tabular}

\begin{tabular}{|l|c|c|c|}
\hline 0.3633 & 0.6785 & 285.26 & 0.05 \\
\hline 0.3869 & 0.7000 & 285.29 & 0.05 \\
\hline 0.4450 & 0.7478 & 284.86 & 0.05 \\
\hline
\end{tabular}

\begin{tabular}{|l|l|l|l|}
\hline 0.4450 & 0.7478 & 284.86 & 0.05 \\
\hline 0.4623 & 0.7607 & 284.70 & 0.05 \\
\hline 0.5167 & 0.7981 & 283.18 & 0.05 \\
\hline
\end{tabular}

\begin{tabular}{|c|c|c|c|}
\hline 0.5167 & 0.7981 & 283.18 & 0.05 \\
\hline 0.5509 & 0.8193 & 281.92 & 0.05 \\
\hline 0.6235 & 0.8596 & 276.83 & 0.05 \\
\hline 0.7706 & 0.9255 & 259.32 & 0.05 \\
\hline 0.8364 & 0.9497 & $256.45 * *$ SLE & -- \\
\hline 0.8455 & 0.9529 & $256.45 * *$ SLE & -- \\
\hline
\end{tabular}

\begin{tabular}{|c|c|c|c|}
\hline \multicolumn{4}{|c|}{ ethanol + n-tridecane } \\
\hline$w_{\mathrm{Et}}$ & $x_{\mathrm{Et}}$ & $T_{\text {cloud }} / \mathrm{K}$ & $\delta T / \mathrm{K}$ \\
\hline 0.0700 & 0.2315 & 275.21 & 0.05 \\
\hline 0.0706 & 0.2330 & 275.03 & 0.08 \\
\hline
\end{tabular}




\begin{tabular}{|c|c|c|c|}
\hline 0.0856 & 0.2725 & 279.15 & 0.14 \\
\hline 0.0940 & 0.2934 & 280.80 & 0.65 \\
\hline 0.0977 & 0.3022 & 280.07 & 0.05 \\
\hline 0.1155 & 0.3431 & 285.80 & 1.41 \\
\hline 0.1406 & 0.3957 & 288.80 & 0.2 \\
\hline 0.1442 & 0.4028 & 287.83 & 0.05 \\
\hline 0.1638 & 0.4395 & 290.99 & 0.11 \\
\hline 0.1718 & 0.4536 & 290.86 & 0.05 \\
\hline 0.1818 & 0.4706 & 293.06 & 0.06 \\
\hline 0.2352 & 0.5517 & 294.59 & 0.05 \\
\hline 0.2486 & 0.5697 & 295.58 & 0.06 \\
\hline 0.2706 & 0.5976 & 295.60 & 0.05 \\
\hline 0.2935 & 0.6244 & 296.86 & 0.05 \\
\hline 0.3167 & 0.6498 & 296.05 & 0.05 \\
\hline 0.3369 & 0.6703 & 297.35 & 0.05 \\
\hline 0.3713 & 0.7026 & 296.21 & 0.05 \\
\hline 0.3957 & 0.7238 & 296.74 & 0.05 \\
\hline 0.4429 & 0.7608 & 296.10 & 0.05 \\
\hline 0.4649 & 0.7766 & 296.45 & 0.05 \\
\hline 0.5282 & 0.8175 & 294.80 & 0.05 \\
\hline 0.5313 & 0.8194 & 294.65 & 0.05 \\
\hline 0.6038 & 0.8591 & 291.27 & 0.05 \\
\hline 0.6685 & 0.8897 & 285.54 & 0.05 \\
\hline 0.6886 & 0.8985 & 284.27 & 0.05 \\
\hline 0.7690 & 0.9302 & 270.78 & 0.05 \\
\hline 0.8247 & 0.9496 & 263.99 & 0.05 \\
\hline
\end{tabular}




\begin{tabular}{|c|c|c|c|}
\hline \multicolumn{4}{|c|}{ ethanol $+n$-tetradecane } \\
\hline$w_{\text {Et }}$ & $x_{\mathrm{Et}}$ & $T_{\text {cloud }} / \mathrm{K}$ & $\delta T / \mathrm{K}$ \\
\hline 0.0544 & 0.1986 & 275.35 & 0.05 \\
\hline 0.0899 & 0.2984 & 286.55 & 0.05 \\
\hline 0.1343 & 0.4005 & 295.96 & 0.05 \\
\hline 0.1843 & 0.4932 & 302.33 & 0.05 \\
\hline 0.1881 & 0.4994 & 302.98 & 0.05 \\
\hline 0.1884 & 0.4999 & 302.71 & 0.05 \\
\hline 0.2178 & 0.5452 & 304.80 & 0.05 \\
\hline 0.2584 & 0.6000 & 306.12 & 0.05 \\
\hline 0.2585 & 0.6001 & 306.49 & 0.05 \\
\hline 0.2995 & 0.6480 & 306.99 & 0.05 \\
\hline 0.3001 & 0.6487 & 307.08 & 0.05 \\
\hline 0.3484 & 0.6972 & 307.48 & 0.05 \\
\hline 0.3514 & 0.7000 & 307.33 & 0.05 \\
\hline 0.3702 & 0.7168 & 307.42 & 0.05 \\
\hline 0.3952 & 0.7378 & 307.38 & 0.05 \\
\hline 0.4789 & 0.7983 & 307.03 & 0.05 \\
\hline 0.4824 & 0.8006 & 306.77 & 0.05 \\
\hline 0.5145 & 0.8203 & 306.59 & 0.05 \\
\hline 0.5661 & 0.8489 & 305.05 & 0.05 \\
\hline 0.5871 & 0.8596 & 304.18 & 0.05 \\
\hline 0.6062 & 0.8689 & 303.21 & 0.05 \\
\hline 0.6513 & 0.8894 & 299.88 & 0.05 \\
\hline 0.6764 & 0.9000 & 297.36 & 0.05 \\
\hline 0.6987 & 0.9090 & 294.98 & 0.05 \\
\hline 0.7782 & 0.9379 & 282.44 & 0.05 \\
\hline
\end{tabular}




\begin{tabular}{|l|l|l|l|}
\hline 0.8143 & 0.9497 & 274.60 & 0.05 \\
\hline
\end{tabular}

\begin{tabular}{|c|c|c|c|}
\hline \multicolumn{4}{|c|}{ ethanol $+n$-pentadecane } \\
\hline$w_{\mathrm{Et}}$ & $x_{\mathrm{Et}}$ & $T_{\text {cloud }} / \mathrm{K}$ & $\delta T / \mathrm{K}$ \\
\hline 0.0628 & 0.2361 & 284.86 & 0.16 \\
\hline 0.0680 & 0.2516 & 287.76 & 0.05 \\
\hline 0.0855 & 0.3013 & 293.48 & 0.28 \\
\hline 0.1014 & 0.3424 & 297.15 & 3.00 \\
\hline 0.1227 & 0.3921 & 303.19 & 0.05 \\
\hline 0.1510 & 0.4506 & 307.00 & 0.05 \\
\hline 0.1633 & 0.4736 & 309.83 & 0.05 \\
\hline 0.1933 & 0.5248 & 312.47 & 0.05 \\
\hline 0.2099 & 0.5506 & 316.63 & 0.10 \\
\hline 0.2262 & 0.5741 & 314.30 & 0.05 \\
\hline 0.2700 & 0.6304 & 315.95 & 0.05 \\
\hline 0.2861 & 0.6488 & 316.55 & 0.05 \\
\hline 0.3028 & 0.6670 & 316.44 & 0.05 \\
\hline 0.3304 & 0.6947 & 317.15 & 0.05 \\
\hline 0.3363 & 0.7003 & 317.25 & 0.05 \\
\hline 0.3623 & 0.7237 & 317.23 & 0.03 \\
\hline 0.3908 & 0.7474 & 317.38 & 0.05 \\
\hline 0.3935 & 0.7495 & 317.25 & 0.05 \\
\hline 0.4271 & 0.7746 & 317.21 & 0.05 \\
\hline 0.4425 & 0.7854 & 317.20 & 0.05 \\
\hline 0.4782 & 0.8086 & 316.79 & 0.05 \\
\hline 0.5475 & 0.8480 & 316.25 & 0.10 \\
\hline 0.5523 & 0.8505 & 315.71 & 0.05 \\
\hline
\end{tabular}




\begin{tabular}{|c|c|c|c|}
\hline 0.6006 & 0.8739 & 314.05 & 0.05 \\
\hline 0.6608 & 0.8998 & 309.15 & 1.50 \\
\hline 0.6608 & 0.8998 & 310.88 & 0.50 \\
\hline 0.7137 & 0.9200 & 305.40 & 0.05 \\
\hline 0.7714 & 0.9396 & 295.34 & 0.05 \\
\hline 0.8016 & 0.9491 & 289.36 & 0.10 \\
\hline 0.8035 & 0.9496 & 287.73 & 0.06 \\
\hline
\end{tabular}

\begin{tabular}{|c|c|c|c|}
\hline \multicolumn{4}{|c|}{ ethanol $+n$-hexadecane } \\
\hline$w_{\text {Et }}$ & $x_{\mathrm{Et}}$ & $T_{\text {cloud }} / \mathrm{K}$ & $\delta T / \mathrm{K}$ \\
\hline 0.0803 & 0.3002 & 296.09 & 0.05 \\
\hline 0.1185 & 0.3978 & 307.99 & 0.05 \\
\hline 0.1668 & 0.4960 & 317.25 & 0.10 \\
\hline 0.1691 & 0.5001 & 317.20 & 0.05 \\
\hline 0.1970 & 0.5466 & 320.02 & 0.05 \\
\hline 0.2343 & 0.6006 & 322.66 & 0.05 \\
\hline 0.2713 & 0.6467 & 324.20 & 0.05 \\
\hline 0.2737 & 0.6494 & 323.75 & 0.05 \\
\hline 0.3200 & 0.6982 & 324.82 & 0.05 \\
\hline 0.3221 & 0.7002 & 325.28 & 0.05 \\
\hline 0.3595 & 0.7340 & 325.72 & 0.05 \\
\hline 0.3903 & 0.7588 & 325.38 & 0.05 \\
\hline 0.4414 & 0.7953 & 325.60 & 0.05 \\
\hline 0.4481 & 0.7996 & 325.52 & 0.05 \\
\hline 0.5122 & 0.8377 & 324.62 & 0.05 \\
\hline 0.5526 & 0.8586 & 324.55 & 0.05 \\
\hline 0.6192 & 0.8888 & 322.24 & 0.05 \\
\hline
\end{tabular}




\begin{tabular}{|l|l|l|l|}
\hline 0.6204 & 0.8893 & 322.41 & 0.05 \\
\hline 0.6469 & 0.9001 & 321.24 & 0.05 \\
\hline 0.6705 & 0.9091 & 319.41 & 0.05 \\
\hline 0.7288 & 0.9296 & 313.79 & 0.10 \\
\hline 0.8290 & 0.9597 & 294.77 & 0.05 \\
\hline
\end{tabular}

\begin{tabular}{|c|c|c|c|}
\hline \multicolumn{4}{|c|}{ ethanol $+n$-heptadecane } \\
\hline$w_{\mathrm{Et}}$ & $x_{\mathrm{Et}}$ & $T_{\text {cloud }} / \mathrm{K}$ & $\delta T / \mathrm{K}$ \\
\hline 0.0790 & 0.3093 & 307.98 & 0.05 \\
\hline 0.0870 & 0.3322 & 310.99 & 0.25 \\
\hline 0.0953 & 0.3547 & 309.03 & 0.05 \\
\hline 0.1102 & 0.3926 & 315.11 & 0.05 \\
\hline 0.1143 & 0.4024 & 314.15 & 0.40 \\
\hline 0.1333 & 0.4452 & 317.70 & 0.50 \\
\hline 0.1347 & 0.4482 & 320.99 & 0.05 \\
\hline 0.1580 & 0.4948 & 324.02 & 0.05 \\
\hline 0.1592 & 0.4972 & 325.05 & 0.40 \\
\hline 0.1744 & 0.5245 & 326.45 & 0.05 \\
\hline 0.2212 & 0.5972 & 328.15 & 0.50 \\
\hline 0.2218 & 0.5980 & 329.32 & 0.05 \\
\hline 0.2612 & 0.6486 & 330.98 & 0.05 \\
\hline 0.2653 & 0.6534 & 330.85 & 0.15 \\
\hline 0.3082 & 0.6993 & 332.55 & 0.50 \\
\hline 0.3639 & 0.7491 & 332.65 & 0.50 \\
\hline 0.3966 & 0.7743 & 333.17 & 0.05 \\
\hline 0.4010 & 0.7775 & 332.65 & 0.50 \\
\hline
\end{tabular}




\begin{tabular}{|l|l|l|l|}
\hline 0.4312 & 0.7983 & 332.97 & 0.05 \\
\hline 0.4326 & 0.7992 & 332.65 & 0.50 \\
\hline 0.4750 & 0.8253 & 332.93 & 0.05 \\
\hline 0.5188 & 0.8491 & 332.67 & 0.05 \\
\hline 0.5198 & 0.8496 & 332.65 & 0.50 \\
\hline 0.5700 & 0.8737 & 332.12 & 0.05 \\
\hline 0.5915 & 0.8831 & 332.07 & 0.05 \\
\hline 0.6313 & 0.8994 & 329.90 & 0.25 \\
\hline 0.6999 & 0.9241 & 326.76 & 0.05 \\
\hline 0.7289 & 0.9335 & 320.50 & 0.05 \\
\hline 0.7821 & 0.9493 & 315.50 & 0.25 \\
\hline 0.7826 & 0.9495 & 315.12 & 0.05 \\
\hline
\end{tabular}

\begin{tabular}{|c|c|c|c|}
\hline \multicolumn{4}{|c|}{ ethanol + n-octadecane } \\
\hline$w_{\mathrm{Et}}$ & $x_{\mathrm{Et}}$ & $T_{\text {cloud }} / \mathrm{K}$ & $\delta T / \mathrm{K}$ \\
\hline 0.0797 & 0.3235 & 308.53 & 0.05 \\
\hline 0.1066 & 0.3972 & 318.77 & 0.05 \\
\hline 0.1515 & 0.4966 & 328.87 & 0.05 \\
\hline 0.1958 & 0.5736 & 334.78 & 0.05 \\
\hline 0.2495 & 0.6474 & 338.38 & 0.05 \\
\hline 0.3315 & 0.7326 & 341.50 & 0.05 \\
\hline 0.3518 & 0.7499 & 341.69 & 0.05 \\
\hline 0.3833 & 0.7744 & 341.56 & 0.05 \\
\hline 0.4197 & 0.7998 & 341.63 & 0.05 \\
\hline 0.4583 & 0.8238 & 341.68 & 0.05 \\
\hline 0.5052 & 0.8494 & 341.63 & 0.05 \\
\hline
\end{tabular}




\begin{tabular}{|l|l|l|l|}
\hline 0.5562 & 0.8738 & 341.15 & 0.05 \\
\hline 0.5901 & 0.8883 & 340.92 & 0.05 \\
\hline 0.6440 & 0.9090 & 338.95 & 0.05 \\
\hline 0.7372 & 0.9394 & 333.24 & 0.05 \\
\hline 0.7912 & 0.9544 & 324.03 & 0.05 \\
\hline 0.8968 & 0.9796 & 298.58 & 0.05 \\
\hline
\end{tabular}

\begin{tabular}{|c|c|c|c|}
\hline \multicolumn{4}{|c|}{ ethanol $+n$-eicosane } \\
\hline$w_{\mathrm{Et}}$ & $x_{\mathrm{Et}}$ & $T_{\text {cloud }} / \mathrm{K}$ & $\delta T / \mathrm{K}$ \\
\hline 0.0543 & 0.2603 & $308.46^{* * \mathrm{SLE}}$ & 0.1 \\
\hline 0.0824 & 0.3552 & 322.13 & 0.05 \\
\hline 0.1034 & 0.4143 & 327.65 & 0.05 \\
\hline 0.1224 & 0.4610 & 334.83 & 0.05 \\
\hline 0.1524 & 0.5244 & 341.54 & 0.05 \\
\hline 0.1974 & 0.6014 & 348.83 & 0.05 \\
\hline 0.2647 & 0.6883 & 352.78 & 0.05 \\
\hline 0.3656 & 0.7795 & 355.82 & 0.05 \\
\hline 0.4796 & 0.8497 & 356.52 & 0.05 \\
\hline 0.5204 & 0.8694 & 356.52 & 0.05 \\
\hline 0.5670 & 0.8893 & 356.32 & 0.05 \\
\hline 0.6193 & 0.9089 & 354.62 & 0.05 \\
\hline 0.7361 & 0.9448 & 350.21 & 0.05 \\
\hline 0.8262 & 0.9668 & 336.80 & 0.05 \\
\hline 0.8529 & 0.9726 & 329.33 & 0.05 \\
\hline 0.8731 & 0.9768 & 323.64 & 0.05 \\
\hline
\end{tabular}




\begin{tabular}{|c|c|c|c|}
\hline \multicolumn{4}{|c|}{ ethanol + n-docosane } \\
\hline$w_{\mathrm{Et}}$ & $x_{\mathrm{Et}}$ & $T_{\text {cloud }} / \mathrm{K}$ & $\delta T / \mathrm{K}$ \\
\hline 0.0993 & 0.4265 & 333.66 & 0.05 \\
\hline 0.1545 & 0.5520 & 349.84 & 0.05 \\
\hline 0.2358 & 0.6754 & 359.78 & 0.05 \\
\hline 0.3836 & 0.8075 & 364.92 & 0.05 \\
\hline 0.4011 & 0.8187 & 365.01 & 0.05 \\
\hline 0.5061 & 0.8736 & 365.16 & 0.05 \\
\hline 0.5954 & 0.9084 & 365.07 & 0.05 \\
\hline 0.7170 & 0.9447 & 362.38 & 0.05 \\
\hline 0.7792 & 0.9597 & 357.76 & 0.05 \\
\hline 0.8507 & 0.9746 & 344.70 & 0.05 \\
\hline
\end{tabular}

\begin{tabular}{|c|c|c|c|}
\hline \multicolumn{4}{|c|}{ ethanol + n-tetracosane } \\
\hline$w_{\mathrm{Et}}$ & $x_{\mathrm{Et}}$ & $T_{\text {cloud }} / \mathrm{K}$ & $\delta T / \mathrm{K}$ \\
\hline 0.0977 & 0.4432 & 343.02 & 0.05 \\
\hline 0.1548 & 0.5739 & 356.14 & 0.05 \\
\hline 0.2504 & 0.7106 & 369.70 & 0.05 \\
\hline 0.4543 & 0.8596 & 374.21 & 0.05 \\
\hline 0.5228 & 0.8895 & 374.55 & 0.05 \\
\hline 0.6249 & 0.9245 & 374.16 & 0.05 \\
\hline 0.7789 & 0.9628 & 367.33 & 0.05 \\
\hline 0.8404 & 0.9748 & 359.57 & 0.05 \\
\hline 0.8983 & 0.9848 & 343.23 & 0.05 \\
\hline
\end{tabular}




\section{References (excerpt):}

62. Dahlmann, U.; Schneider, G. M., (Liquid + Liquid) Phase Equilibria and Critical Curves of (Ethanol + Dodecane or Tetradecane or Hexadecane or 2,2,4,4,6,8,8Heptamethylnonane) from 0.1 MPa to 120.0 MPa. J. Chem. Thermodyn. 1989, 21, 997-1004. 63. Peleteiro, J.; Troncoso, J.; Gonzalez-Salgado, D.; Valencia, J. L.; Cerdeirina, C. A.; Romani, L., Anomalous Excess Heat Capacities of Ethanol + Alkane Mixtures. Int. J. Thermophys. 2004, 25, 787-803.

64. Matsuda, H.; Ochi, K., Liquid-Liquid Equilibrium Data for Binary Alcohol plus nAlkane (C10-C16) Systems: Methanol plus Decane, Ethanol plus Tetradecane, and Ethanol plus Hexadecane. Fluid Phase Equilib. 2004, 224, 31-37.

65. French, H. T.; Richards, A.; Stokes, R. H., Thermodynamics of the Partially Miscible system Ethanol + Hexadecane. J. Chem. Thermodyn. 1979, 11, 671-686.

66. Chang, S. S.; Maurey, J. R.; Pummer, W. J., Solubilities of two $n$-Alkanes in various Solvents. J. Chem. Eng. Data 1983, 28, 187-189. 